Research Article

\title{
Performance Evaluation of Cognitive Radio Networks with Imperfect Spectrum Sensing and Bursty Primary User Traffic
}

\author{
Osama Salameh, ${ }^{1,2}$ Herwig Bruneel, ${ }^{1}$ and Sabine Wittevrongel $\mathbb{D}^{1}$ \\ ${ }^{1}$ Department of Telecommunications and Information Processing (TELIN), Ghent University, Sint-Pietersnieuwstraat 41, \\ Gent B-9000, Belgium \\ ${ }^{2}$ Faculty of Engineering and Information Technology, Arab American University, P.O. Box 240, Jenin, State of Palestine
}

Correspondence should be addressed to Sabine Wittevrongel; sabine.wittevrongel@ugent.be

Received 19 September 2019; Accepted 7 May 2020; Published 2 June 2020

Academic Editor: Xiao-Qiao He

Copyright (c) 2020 Osama Salameh et al. This is an open access article distributed under the Creative Commons Attribution License, which permits unrestricted use, distribution, and reproduction in any medium, provided the original work is properly cited.

\begin{abstract}
In this paper, we introduce a four-dimensional continuous-time Markov chain model to evaluate the performance of cognitive radio networks. In such networks, secondary (unlicensed) users may opportunistically use the frequency channels not currently occupied by primary (licensed) users in order to increase the utilization of the wireless spectrum. Secondary users perform channel sensing before as well as during transmission in order not to interfere with primary users. The proposed model assumes that primary users arrive according to a bursty arrival process and moreover takes the possible occurrence of sensing errors (false alarms and misdetections) into account. Several performance measures including the collision rate between primary and secondary users, the blocking probabilities of primary or secondary users, and the mean delay of secondary users are derived and illustrated through numerical examples. The results show that the system performance strongly depends on the degree of burstiness in the arrival process of primary users. It is also observed that the quality of service of the primary network can be seriously compromised due to misdetection by secondary users.
\end{abstract}

\section{Introduction}

Cognitive radio networks (CRNs) are an efficient paradigm to address the scarcity of the wireless spectrum $[1,2]$. Under this paradigm, two types of networks operate simultaneously. The first type is a primary network that owns the spectrum and coordinates the access of primary users (PUs) to individual channels. The second type is a secondary network where secondary users (SUs) are allowed to make use of the channels not occupied by PUs. The sole purpose of the secondary network is to increase the utilization of the underutilized spectrum. We assume that both networks operate independently of each other and that the SUs have no knowledge of the access patterns of the PUs. Whenever a SU needs to transmit, it must perform channel sensing to ensure that a channel is idle. Also, a SU must perform sensing while transmitting in order to avoid collision with a possible arrival of a PU. A comparison of different sensing techniques is available in $[3,4]$.

In practice, two kinds of SU sensing errors are common: false alarm and misdetection of a PU. In the event of a false alarm, a SU incorrectly detects the presence of a PU on a channel, which results in a lost transmission opportunity. In case of a misdetection of a PU, a SU fails to detect the actual presence of a PU, which results in a collision between the PU and the SU and the loss of both users. Both kinds of SU sensing errors can occur before and during a SU transmission. As a consequence and following [5], we distinguish two types of false alarm and two classes of misdetection. Type-I false alarm occurs when a sensing SU is unable to detect an idle channel, and Type-II false alarm occurs when a transmitting SU evacuates the channel without PU arrival. Class-A misdetection occurs when a sensing SU incorrectly decides that a channel is idle; when in reality, it is occupied 
by a PU, and class-B misdetection occurs when a transmitting SU fails to detect the arrival of a PU on the channel. In this paper, both kinds of sensing errors are considered.

Most of the results of the research on CRNs obtained so far are based on the assumption that the PU network is timeslotted, and the SU network is perfectly synchronized with the primary network as, e.g., in [6-11]. While the synchronization between SU and PU networks can be difficult to implement as it may require the SU to know the details of the PU network like the exact slot structure, some of the PU networks are not time-slotted. In this paper, we therefore investigate the performance of CRNs where both PU and SU networks are not time-slotted. The problems that emerge from such assumptions are viewed as a new research direction [12]. Furthermore, the majority of works reported in the literature assumes that PUs arrive according to a Poisson process. This model may not be accurate for PUs in a practical environment $[13,14]$, which in turn affects the accuracy of the obtained performance measures. Incorporating more realistic models in the context of cognitive radio is reported as a research challenge [15], to be considered for the future work [12], where the interrupted Poisson process (IPP) is explicitly mentioned as an example. To fill this gap, we study in this paper the performance of a CRN with asynchronous interaction between SUs and PUs, and our aim is to gain insight into the CRN performance with more realistic (bursty) traffic models of PUs. In particular, we model the arrival process of PUs as an IPP. This model has been reported suitable for web browsing [16] or data traffic [17].

Our main contribution in the present paper is the analysis of an analytic model of a CRN under bursty PU traffic and including SU sensing errors. In particular, we introduce a four-dimensional continuous-time Markov chain (CTMC) model to evaluate the CRN performance under bursty PU arrivals and SU sensing errors. To the best of our knowledge, such a model is not available in the literature. For further background on the theory of stochastic processes and CTMCs, we refer to [18-20].

The rest of this paper is organized as follows. In Section 2 , the related work and our contribution are discussed. In Section 3, the system model is presented, and in Section 4, the related CTMC model is derived. We formalize the performance measures in Section 5, and we discuss some numerical results in Section 6. In Section 7, the conclusion is given.

\section{Related Works and Contribution}

In this section, we review some of the studies that assume asynchronous interaction between SUs and PUs and use a CTMC modeling approach. For a summary of our comparison, we refer to Table 1. In [21], a CRN comprising one PU and $N$ SUs was studied, where the whole spectrum is treated as one channel exclusively allocated to one user at a time. A steady-state analysis is conducted. In [22], Markov models were developed to study a multichannel CRN. Several performance measures are derived including the mean delay of the SUs, the variance of the SU delay, and the interruption probability of SUs by PU arrival. Both works assume that SUs have perfect sensing abilities, and the presence of SUs does not degrade the performance of the primary network, which is not always true in practice. In [23], the authors analyzed a system where both PUs and SUs access the spectrum through a PU base station (PU-BS). SUs have sensing abilities, and an arriving SU accesses the PU-BS with a certain probability $\beta$ based on the current system load. A transmitting SU may be dropped with probability $\alpha$ upon the arrival of a PU when all the channels are busy. This paper also considers a collision-free environment between PUs and SUs, but the performance of the PUs may get affected by the presence of SUs when all the channels are busy.

An analysis of a CRN with imperfect sensing is conducted in [24-27]. The PU termination probability due to collision with SUs is obtained in [24]. This analysis is conducted for the case where the number of channels $N=3$. A limitation of this work is the assumption that a transmitting SU will always evacuate the channel upon PU arrival, i.e., no misdetection of an arriving PU is considered. Other limitations are that the SU sensing time is considered negligible, and false alarms of transmitting SUs are not taken into consideration. In $[25,26]$, different extensions of the study of [24] were reported, where new performance measures were investigated. The restriction on the number of channels is removed in $[25,26]$, but all other limitations of [24] mentioned above still hold. In [27], the authors investigated the case where the SUs are able to sense all the channels in the system to determine the access probability. The blocking probability of PUs and the probability of a SU being dropped are derived. It is important to note that sensing errors of transmitting SUs are not taken into consideration in all the studies [24-27].

Sensing errors of sensing SUs and transmitting SUs are considered in $[5,28]$. In [28], the case when a colliding PU and SU are dropped from the system was analyzed. In [5], the analysis was extended to other cases when only one of the colliding users is dropped if a collision occurs. The limitations of these studies include the absence of SU sensing time and the absence of SU handoff time, where the latter is the time needed for a transmitting SU to switch to another channel upon the arrival of a PU. The effect of interruption of a transmitting SU by an arriving PU is only considered when no idle channels are available. Furthermore, these studies assume that collisions are not influenced by the number of transmitting SUs in the system. It is worth mentioning that all the studies reviewed in this section are based on an appropriate CTMC, and the arrival of PUs is modeled as a Poisson process. Our previous study [29] is free of all the limitations mentioned above, except that it also models the PU arrival by means of a Poisson process.

Our present paper is an extension of [29] in the sense that PUs arrive according to an IPP. Our objective is to analyze CRNs with imperfect SU sensing and bursty PU traffic using a CTMC model in terms of a number of performance measures. We evaluate SU-related performance measures including the delay of SUs, blocking probability of SUs, and throughput rate of SUs. Also, we evaluate measures related to the $\mathrm{PU}$ performance, such as the blocking 
TABLE 1: Comparison among various CTMC-based models.

\begin{tabular}{lcccc}
\hline Reference no. & Sensing time & Arriving SU errors & Transmitting SU errors & Bursty PU arrival (IPP) \\
\hline$[21,23]$ & $\boldsymbol{X}$ & $\checkmark$ & $\boldsymbol{X}$ & $\boldsymbol{X}$ \\
{$[22]$} & $\checkmark$ & $\boldsymbol{X}$ & $\boldsymbol{X}$ & $\boldsymbol{X}$ \\
{$[24-27]$} & $\boldsymbol{X}$ & $\checkmark$ & $\checkmark$ & $\boldsymbol{X}$ \\
{$[5,28]$} & $\boldsymbol{V}$ & $\checkmark$ & $\checkmark$ & $\boldsymbol{X}$ \\
{$[29]$} & $\checkmark$ & $\checkmark$ & $\checkmark$ & $\sqrt{ }$ \\
Proposed model & $\checkmark$ & $\checkmark$ & & \\
\hline
\end{tabular}

probability of PUs and the collision rate between PUs and SUs affecting both networks. Closed-form expressions for the calculation of all these measures are derived in terms of the steady-state distribution of the involved CTMC.

\section{System under Study and Modeling Assumptions}

We consider a CRN where primary and secondary user networks operate in parallel, as illustrated in Figure 1. In this section, we describe the operation of both networks and the related modeling assumptions. The system parameters are summarized in Table 2.

3.1. Primary User Network. In the primary network, PUs share $N$ frequency bands (channels). Arriving PUs are ready to transmit data, and their access to the channels is coordinated by a primary base station such that the primary network is collision-free, i.e., no collisions between PUs can occur. In particular, an arriving PU is randomly assigned an available channel, i.e., a channel not already occupied by another PU. If there are no available channels, the arriving PU is blocked. Note that the primary network has no knowledge about the SU activity, and therefore, all channels occupied by SUs are considered as available channels, and PUs can be assigned to them (the transmitting SU will need to evacuate the channel in such a case). All channels have the same bandwidth and are not time-slotted. Moreover, the frequency channels are error-free, i.e., if a PU starts transmission, it is considered successful unless there is a collision with a SU during transmission due to a misdetection (see below). The PU transmission duration (or channel holding time) is assumed to be exponentially distributed with rate $\mu_{1}$, i.e., with mean value $1 / \mu_{1}$.

The arrival of PUs is modeled by an interrupted Poisson process (IPP). This is a two-state Markovian process with an active and an inactive state, as shown in Figure 2. In the active state, PUs arrive in accordance with a Poisson process with rate $\lambda_{1}^{*}$, whereas no PUs arrive in the inactive state. The active and inactive periods are both exponentially distributed. We let $\gamma_{1}$ denote the rate from the inactive to the active state and $\gamma_{2}$ the rate from the active to the inactive state. Then, the steadystate probability that the IPP is in the active state is given by

$$
P_{\text {active }}=\frac{\gamma_{1}}{\gamma_{1}+\gamma_{2}}
$$

and the average PU arrival rate equals $\lambda_{1}=\lambda_{1}^{*} P_{\text {active }}$. The mean active and inactive periods are given by $1 / \gamma_{2}$ and $1 / \gamma_{1}$,

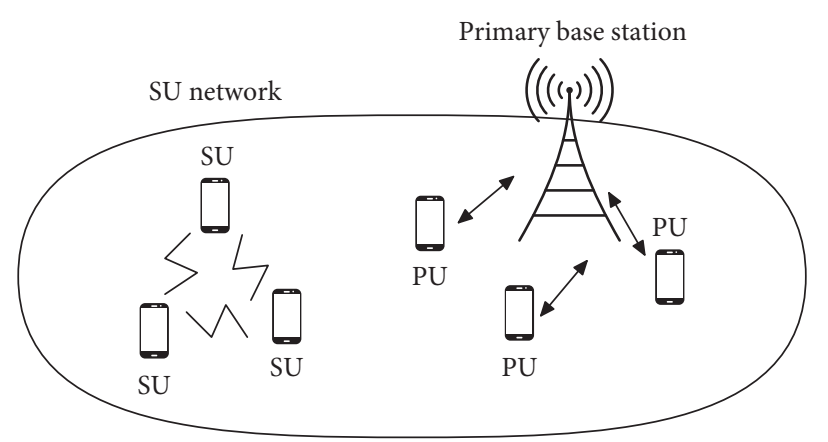

FIgURE 1: Cognitive radio network environment with primary and secondary user networks.

TABle 2: Cognitive radio system parameters.

\begin{tabular}{lc}
\hline$N$ & Number of frequency channels \\
$\lambda_{1}^{*}$ & PU arrival rate in active state \\
$1 / \gamma_{1}$ & Mean inactive period of IPP for PU arrivals \\
$1 / \gamma_{2}$ & Mean active period of IPP for PU arrivals \\
$\lambda_{1}$ & Average PU arrival rate \\
$\lambda_{2}$ & SU arrival rate \\
$\mu_{1}$ & PU transmission rate \\
$\mu_{2}$ & SU transmission rate \\
$K$ & Maximum number of sensing SUs \\
$\sigma$ & Channel sensing rate \\
$p_{m 1}$ & Class-A misdetection probability \\
$p_{m 2}$ & Class-B misdetection probability \\
$p_{f 1}$ & Type-I false alarm probability \\
$\delta_{f 2}$ & Type-II false alarm rate \\
\hline
\end{tabular}

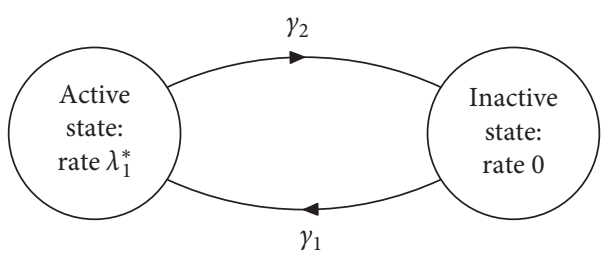

FIgURE 2: Interrupted Poisson process model.

respectively. For a given value of $P_{\text {active }}$, i.e., a given ratio $\gamma_{1} / \gamma_{2}$, smaller values of $\gamma_{1}$ and $\gamma_{2}$ correspond to longer active and inactive periods and hence a more bursty PU arrival process.

3.2. Secondary User Network. SUs are allowed to make use of licensed channels that are temporarily not occupied by PUs. The secondary network is an infrastructureless ad hoc 
wireless network. There is no central entity like a base station to coordinate the channel access by SUs and avoid interference with PUs. On the contrary, each SU is equipped with all the cognitive capabilities to sense the spectrum and to determine if a channel is occupied by a PU or idle based on its own measurements. SUs are also aware of the channels occupied by other SUs by the exchange of messages among SUs, as also indicated in, e.g., $[5,28,30]$, so no collisions between SUs can occur. The SU network is not time-slotted. SUs not only sense the spectrum upon arrival in order to find an idle channel for transmission but also during transmission to avoid collision with arriving PUs. With respect to the latter, we assume that SUs operate in full duplex mode, i.e., a SU can sense and transmit simultaneously, as also considered in [30-33]. This allows an efficient evacuation of the channel by the SU upon arrival of a PU on the channel.

We assume in our model that SUs arrive according to a Poisson process with rate $\lambda_{2}$. Several admission control policies of SUs are used in CRNs. These policies aim at ensuring quality of service for both SUs and PUs [34] and are implemented by limiting the access of users to the system [35]. We consider the case where an arriving SU starts sensing to find an idle channel if the number of sensing SUs in the system is below a given number $K$ and gets blocked, otherwise. We refer to the maximum number $K$ of sensing SUs as the sensing room size. Once admitted to the sensing room, a SU randomly selects a channel from the set of idle channels or those occupied by PUs and senses the channel. The channel sensing time is assumed to be exponentially distributed with rate $\sigma$ for mathematical tractability; such assumption of nonfixed sensing time has been used before in, e.g., [36]. With respect to the outcome of the sensing of a channel by a $\mathrm{SU}$, the following options are possible:

(i) The SU correctly detects PU presence on the channel. The SU then picks another channel and starts to sense again.

(ii) The SU perceives the channel as occupied by a PU while it actually is idle, so the SU fires a type-I false alarm. We denote the type-I false alarm probability by $p_{f 1}$, i.e., $p_{f 1}$ is the probability that a $S U$ who has finished sensing an idle channel erroneously thinks a PU is occupying the channel. Also, in this case, the SU picks another channel and starts to sense again.

(iii) The SU correctly detects an idle channel and starts transmission on that channel.

(iv) The SU perceives the channel as idle while it in fact is occupied by a PU, which corresponds to a class-A misdetection. We let $p_{m 1}$ denote the class-A misdetection probability. In this case, there is a collision between the $\mathrm{SU}$ and the PU, and both users are dropped from the system.

A SU transmits on a single frequency channel, and we assume that the SU transmission duration is exponentially distributed with rate $\mu_{2}$. While transmitting, the SU continuously senses its channel in order to detect the possible arrival of a PU. The following events may then occur instantaneously during the sensing: (i) A PU arrives and the transmitting SU correctly detects its arrival. The SU then evacuates the channel and starts sensing to find an idle channel provided the sensing room is not full or gets lost, otherwise.

(ii) The transmitting SU incorrectly records PU arrival and thus fires a type-II false alarm. We denote the rate of firing a type-II false alarm by $\delta_{f 2}$. Likewise, in this case, the SU stops its transmission and either starts sensing for an idle channel in case of a nonfull sensing room or gets lost, otherwise.

(iii) The transmitting SU misdetects PU arrival on the channel. The SU and the PU will then collide and both be dropped from the system. This corresponds to a class- $\mathrm{B}$ misdetection, and the probability of such misdetection is denoted by $p_{m 2}$.

Note that, as long as none of the above 3 events occurs during the sensing, the SU will simply continue its transmission until it is completed. Also, note that the occurrence of type-II false alarms is modeled by the rate $\delta_{f 2}$, whereas the other error parameters $p_{f 1}, p_{m 1}$, and $p_{m 2}$ are probabilities. This is because a SU can fire a type-II false alarm at any time instant during transmission, whereas the other sensing errors are tied to specific instants. Specifically, the parameters $p_{m 1}$ and $p_{f 1}$ are related to the instant when a SU finishes sensing a channel, while $p_{m 2}$ is related to the instant of arrival of a PU to a channel.

\section{Continuous-Time Markov Chain Model}

Given the above modeling assumptions, the performance of the considered system can be investigated by means of a four-dimensional continuous-time Markov chain (CTMC) model. In particular, we define system state $x$ as the quadruplet $x=\left(x_{1}, x_{2}, x_{3}, x_{4}\right)$, where $x_{1}$ denotes the number of PUs in the system, $x_{2}$ and $x_{3}$ are the numbers of transmitting and sensing SUs, respectively, and $x_{4}$ indicates whether the PU arrival process is in the active state $\left(x_{4}=1\right)$ or in the inactive state $\left(x_{4}=0\right)$. The finite state space $S$ of the CTMC contains all states $\left(x_{1}, x_{2}, x_{3}, x_{4}\right)$ satisfying the conditions

$$
x_{1}+x_{2} \leq N, x_{3} \leq K \text {, and } x_{4}=0,1 \text {. }
$$

In the rest of this section, we focus on the derivation of the infinitesimal generator $Q$ of the CTMC. On the one hand, it is a well-known property that the row sums of this matrix are equal to zero such that the diagonal element $q_{x, x}$ of $Q(x \in S)$ is expressed as

$$
q_{x, x}=-\sum_{y \in S, y \neq x} q_{x, y} .
$$

On the other hand, the nondiagonal element $q_{x, y}(x \neq y)$ of the matrix $Q$ is the total transition rate from state $x$ to another state $y$. To obtain $q_{x, y}(x \neq y)$, we therefore need to list all events that cause a change from system state $x=$ $\left(x_{1}, x_{2}, x_{3}, x_{4}\right)$ to another state $y$ and to determine the corresponding transition rates.

A first event is related to PU arrival, which occurs with rate $\lambda_{1}^{*}$ if the IPP process of PUs is in the active state, i.e., if 
$x_{4}=1$. Such PU arrival will cause a change in the system state if there are still available channels for PUs, i.e., if $x_{1}<N$, and one of the $N-x_{1}$ available channels will then randomly be assigned to the arriving PU. In case the assigned channel is an idle channel, the number of PUs in the system simply increases by one and we get a transition to state $y=\left(x_{1}+1, x_{2}, x_{3}, x_{4}\right)$. In case the assigned channel is already in use by a transmitting SU and the PU arrival is correctly detected by the transmitting SU (i.e., there is no
class-B misdetection), the SU will moreover evacuate the channel and start to sense again if $x_{3}<K$ or leave the system if $x_{3}=K$. Finally, in case of an assigned channel already occupied by a SU together with a class- $\mathrm{B}$ misdetection, a collision occurs between the arriving PU and the transmitting SU, and both users are dropped from the system such that the system state changes to $y=\left(x_{1}, x_{2}-1, x_{3}, x_{4}\right)$. These observations lead to the following transition equations related to PU arrival:

$$
\begin{aligned}
& q_{x, y}=\lambda_{1}^{*} \frac{N-x_{1}-x_{2}}{N-x_{1}}, \quad \text { if } y=\left(x_{1}+1, x_{2}, x_{3}, x_{4}\right), x_{1}<N, x_{4}=1, \\
& q_{x, y}=\lambda_{1}^{*} \frac{x_{2}}{N-x_{1}}\left(1-p_{m 2}\right), \quad \text { if } y=\left(x_{1}+1, x_{2}-1, x_{3}+1, x_{4}\right), x_{1}<N, x_{4}=1, x_{3}<K, \\
& q_{x, y}=\lambda_{1}^{*} \frac{x_{2}}{N-x_{1}}\left(1-p_{m 2}\right), \quad \text { if } y=\left(x_{1}+1, x_{2}-1, x_{3}, x_{4}\right), x_{1}<N, x_{4}=1, x_{3}=K .
\end{aligned}
$$

Note that the case of a class-B misdetection is not included in equations (4)-(6); since also other events may cause a transition to state $y=\left(x_{1}, x_{2}-1, x_{3}, x_{4}\right)$, the expression for the total transition rate to this state is postponed until later.

A second event similarly corresponds to SU arrival, which occurs with rate $\lambda_{2}$ and causes a state change if the number of sensing SUs already in the system is less than $K$. This leads to

$$
q_{x, y}=\lambda_{2}, \quad \text { if } y=\left(x_{1}, x_{2}, x_{3}+1, x_{4}\right), x_{3}<K .
$$

Next, each of the $x_{1}$ transmitting PUs can complete transmission with rate $\mu_{1}$ such that

$$
q_{x, y}=x_{1} \mu_{1}, \quad \text { if } y=\left(x_{1}-1, x_{2}, x_{3}, x_{4}\right) .
$$

Similarly, a SU transmission completion occurs with rate $x_{2} \mu_{2}$, which changes the system state to state $y=\left(x_{1}, x_{2}-1, x_{3}, x_{4}\right)$. Moreover, a type-II false alarm occurs with rate $x_{2} \delta_{f 2}$, and such a false alarm makes the corresponding transmitting SU stop its transmission and return to the sensing state if $x_{3}<K$ or leave the system if $x_{3}=K$. Taking into account that a change to state $y=\left(x_{1}, x_{2}-1, x_{3}, x_{4}\right)$ may also be caused by PU arrival together with a class-B misdetection, the next set of transition equations we get is as follows:

$$
\begin{aligned}
& q_{x, y}=x_{2} \mu_{2}, \quad \text { if } y=\left(x_{1}, x_{2}-1, x_{3}, x_{4}\right), x_{4}=0, x_{3}<K, \\
& q_{x, y}=x_{2}\left(\mu_{2}+\delta_{f 2}\right), \quad \text { if } y=\left(x_{1}, x_{2}-1, x_{3}, x_{4}\right), x_{4}=0, x_{3}=K, \\
& q_{x, y}=\lambda_{1}^{*} \frac{x_{2}}{N-x_{1}} p_{m 2}+x_{2} \mu_{2}, \quad \text { if } y=\left(x_{1}, x_{2}-1, x_{3}, x_{4}\right), x_{1}<N, x_{4}=1, x_{3}<K, \\
& q_{x, y}=\lambda_{1}^{*} \frac{x_{2}}{N-x_{1}} p_{m 2}+x_{2}\left(\mu_{2}+\delta_{f 2}\right), \quad \text { if } y=\left(x_{1}, x_{2}-1, x_{3}, x_{4}\right), x_{1}<N, x_{4}=1, x_{3}=K, \\
& q_{x, y}=x_{2} \delta_{f 2}, \quad \text { if } y=\left(x_{1}, x_{2}-1, x_{3}+1, x_{4}\right), x_{3}<K .
\end{aligned}
$$

Another event corresponds to a sensing SU finishing the sensing of a channel, which occurs with rate $x_{3} \sigma$, provided that not all channels are already occupied by transmitting SUs, i.e., if $x_{2}<N$. Indeed, since SUs are aware of the channels in use by other SUs, SUs will only sense the $N-x_{2}$ channels not already occupied by other SUs. In case the sensed channel is idle and this is correctly detected by the $\mathrm{SU}$, i.e., there was no type-I false alarm, the SU starts transmission. In case the sensed channel is in use by a PU and this is not correctly detected by the SU, i.e., there was a 
class-A misdetection, the SU starts transmission, and the collided PU and SU are both dropped from the system. The corresponding transition equations are

$$
\begin{aligned}
q_{x, y}= & x_{3} \sigma \frac{N-x_{2}-x_{1}}{N-x_{2}}\left(1-p_{f 1}\right), \\
& \text { if } y=\left(x_{1}, x_{2}+1, x_{3}-1, x_{4}\right), x_{2}<N, \\
q_{x, y}= & x_{3} \sigma \frac{x_{1}}{N-x_{2}} p_{m 1}, \\
& \text { if } y=\left(x_{1}-1, x_{2}, x_{3}-1, x_{4}\right), x_{2}<N .
\end{aligned}
$$

A final set of events that cause a system state change corresponds to state changes in the PU arrival process, which occur with rate $\gamma_{1}$ from the inactive to the active state and rate $\gamma_{2}$ vice versa. We thus have

$$
\begin{array}{ll}
q_{x, y}=\gamma_{1}, & \text { if } y=\left(x_{1}, x_{2}, x_{3}, x_{4}+1\right), x_{4}=0, \\
q_{x, y}=\gamma_{2}, & \text { if } y=\left(x_{1}, x_{2}, x_{3}, x_{4}-1\right), x_{4}=1 .
\end{array}
$$

For all other combinations of state $x$ and $y(x \neq y)$, the transition rate $q_{x, y}$ equals 0 :

$$
q_{x, y}=0, \quad \text { otherwise. }
$$

We now denote by $\pi_{\left(x_{1}, x_{2}, x_{3}, x_{4}\right)}$ the steady-state probability that the system is in state $x=\left(x_{1}, x_{2}, x_{3}, x_{4}\right) \in S$. The row vector $\pi$ with elements $\pi_{\left(x_{1}, x_{2}, x_{3}, x_{4}\right)}$ is then calculated from the set of equilibrium conditions for the CTMC:

$$
\pi Q=0 \text {, }
$$

in combination with the normalization condition $\pi 1=1$, where 1 is a column vector of ones of appropriate size.

\section{Performance Measures}

Based on the vector of steady-state probabilities $\pi$, several performance measures of the cognitive radio system can be computed. In this section, we give expressions for the collision rate between PUs and SUs, the blocking probabilities of PUs and SUs, the throughputs of PUs and SUs, and the mean delay of SUs.

First, the collision rate $\alpha$ between PUs and SUs is the rate at which PUs and SUs run into a collision due to a class-A or class- $B$ misdetection and are dropped from the system. In view of the transition equations (11), (12), and (15), the collision rate $\alpha$ is calculated as follows:

$$
\begin{aligned}
\alpha= & \sum_{x_{2}=0}^{N-1} \sum_{x_{1}=0}^{N-x_{2}} \sum_{x_{3}=0}^{K} \sum_{x_{4}=0}^{1} \pi_{\left(x_{1}, x_{2}, x_{3}, x_{4}\right)} x_{3} \sigma \frac{x_{1}}{N-x_{2}} p_{m 1} \\
& +\sum_{x_{1}=0}^{N-1} \sum_{x_{2}=0}^{N-x_{1}} \sum_{x_{3}=0}^{K} \pi_{\left(x_{1}, x_{2}, x_{3}, 1\right)} \lambda_{1}^{*} \frac{x_{2}}{N-x_{1}} p_{m 2} .
\end{aligned}
$$

Note that, in the first term, the summation runs over all states in the state space $S$ with $x_{2}<N$ since a class-A misdetection can only occur in such states. Similarly, the summation in the second term runs over all states with
$x_{1}<N$ and $x_{4}=1$ since a class-B misdetection is only possible in such states.

Secondly, the blocking probability $\beta_{\mathrm{PU}}$ of PUs is the probability that an arriving PU finds all $N$ channels already occupied by other PUs and therefore gets blocked. Taking into account the IPP arrival process of PUs, the blocking probability $\beta_{\mathrm{PU}}$ is given by

$$
\beta_{\mathrm{PU}}=\frac{1}{P_{\text {active }}} \sum_{x_{3}=0}^{K} \pi\left(N, 0, x_{3}, 1\right) .
$$

The blocking probability $\beta_{\mathrm{SU}}$ of SUs corresponds to the probability that an arriving SU finds already $K$ sensing SUs in the system and therefore equals

$$
\beta_{S U}=\sum_{x_{1}=0}^{N} \sum_{x_{2}=0}^{N-x_{1}} \sum_{x_{4}=0}^{1} \pi_{\left(x_{1}, x_{2}, K, x_{4}\right)} .
$$

Next, the throughput $\eta_{\mathrm{PU}}$ of PUs is the rate at which PUs successfully complete transmission and is hence given by

$$
\eta_{P U}=\sum_{x_{1}=0}^{N} \sum_{x_{2}=0}^{N-x_{1}} \sum_{x_{3}=0}^{K} \sum_{x_{4}=0}^{1} \pi_{\left(x_{1}, x_{2}, x_{3}, x_{4}\right)} x_{1} \mu_{1} .
$$

Similarly, the throughput $\eta_{\mathrm{SU}}$ of SUs is calculated as

$$
\eta_{S U}=\sum_{x_{1}=0}^{N} \sum_{x_{2}=0}^{N-x_{1}} \sum_{x_{3}=0}^{K} \sum_{x_{4}=0}^{1} \pi_{\left(x_{1}, x_{2}, x_{3}, x_{4}\right)} x_{2} \mu_{2} \text {. }
$$

Finally, from Little's law [19], the mean delay $E\left[d_{S U}\right]$ of SUs, i.e., the total time SUs spends in the cognitive radio system between the instant of effectively entering the system and their departure instant from the system, can be obtained as

$$
E\left[d_{S U}\right]=\frac{E\left[n_{S U, t r}\right]+E\left[n_{S U, s e}\right]}{\lambda_{2}\left(1-\beta_{S U}\right)},
$$

where $\lambda_{2}\left(1-\beta_{S U}\right)$ is the effective arrival rate of SUs in the system and $E\left[n_{S U, t r}\right]$ and $E\left[n_{S U, s e}\right]$ are the mean numbers of transmitting and sensing SUs in the system, respectively:

$$
\begin{aligned}
& E\left[n_{S U, t r}\right]=\sum_{x_{1}=0}^{N} \sum_{x_{2}=0}^{N-x_{1}} \sum_{x_{3}=0}^{K} \sum_{x_{4}=0}^{1} x_{2} \pi_{\left(x_{1}, x_{2}, x_{3}, x_{4}\right)}, \\
& E\left[n_{S U, s e}\right]=\sum_{x_{1}=0}^{N} \sum_{x_{2}=0}^{N-x_{1}} \sum_{x_{3}=0}^{K} \sum_{x_{4}=0}^{1} x_{3} \pi_{\left(x_{1}, x_{2}, x_{3}, x_{4}\right)} .
\end{aligned}
$$

\section{Numerical Results}

In this section, we investigate the performance of the considered cognitive radio system by means of several numerical examples. In all examples, the number of frequency channels is $N=20$. We define the PU load as $\rho_{\mathrm{PU}}=$ $\lambda_{1} /\left(\mu_{1} N\right)$ and the SU load as $\rho_{\mathrm{SU}}=\lambda_{2} /\left(\mu_{2} N\right)$. We consider the case where $\rho_{\mathrm{SU}}=0.5$. The transmission durations of the PUs and the SUs both have mean values $1 / \mu_{1}=1 / \mu_{2}=10 \mathrm{~ms}$. We consider a mean SU sensing time 
$1 / \sigma=10 \mathrm{~ms}$, and the maximum number of sensing SUs equals $K=50$.

In the first set of examples (Figures 3-7), we investigate the effect of the IPP parameters $\lambda_{1}^{*}, \gamma_{1}$, and $\gamma_{2}$ representing the PU traffic on the performance measures. We assume that $\gamma_{1}=\gamma_{2}$ which makes $P_{\text {active }}=P_{\text {inactive }}=0.5$. This assumption is close to the approximation of real PU traffic [37]. In this set of examples, we consider the case where SUs have perfect sensing abilities, i.e., where $p_{f 1}=p_{m 1}=p_{m 2}=0$ and $\delta_{f 2}=0 /$ s. In Figures 3-7, we also compare the performance to the case where PUs are assumed to arrive according to a Poisson process with the same average PU arrival rate $\lambda_{1}$. The results for the case of Poisson arrivals are obtained based on our earlier model in [29].

Figure 3 shows the blocking probability $\beta_{\mathrm{PU}}$ of PUs versus the average PU arrival rate $\lambda_{1}$, for different values of $\gamma_{1}=\gamma_{2}$. As expected, for small $\lambda_{1}$, the blocking probability $\beta_{\mathrm{PU}}$ is close to 0 regardless of the value of $\gamma_{1}=\gamma_{2}$. For higher values of $\lambda_{1}$, we observe that the PU blocking probability is higher for lower values of $\gamma_{1}=\gamma_{2}$. This is an expected result since smaller values of $\gamma_{1}=\gamma_{2}$ correspond to longer active and inactive periods and a more bursty PU arrival process. Note that this higher blocking probability for lower $\gamma_{1}=\gamma_{2}$ also means that, for a given value of $\lambda_{1}$, i.e., for a given PU load, the effective arrival rate $\lambda_{1}\left(1-\beta_{\mathrm{PU}}\right)$ of PUs entering the system decreases as $\gamma_{1}=\gamma_{2}$ decreases. From Little's law and in view of the constant PU transmission duration considered in Figure 3, it then immediately follows that, for a given value of $\lambda_{1}$, the mean number of PUs in the system decreases as $\gamma_{1}=\gamma_{2}$ decreases. Finally, Figure 3 shows that the PU blocking probability is lower for the case of a Poisson arrival process than for IPP arrivals. This is intuitively clear since the Poisson process does not take any time correlation in the PU arrival process into account, while under the IPP model, burstiness is not neglected and PUs tend to arrive to the system in a more clustered way.

Figure 4 shows the mean delay of SUs versus the average PU arrival rate $\lambda_{1}$, again for different values of $\gamma_{1}=\gamma_{2}$. When the PU load $\rho_{\mathrm{PU}}$ is small, the effect of $\gamma_{1}=\gamma_{2}$ on the mean SU delay is rather small. In this case, for a fixed PU load, the number of PU packet arrivals during an active period is higher for small $\gamma_{1}=\gamma_{2}$, and the more bursty PU arrival process also causes a slight increase in the number of sensing SUs and the mean SU delay. For higher values of $\lambda_{1}$, more PU packet arrivals are blocked for small values of $\gamma_{1}=\gamma_{2}$ (see also Figure 3), and thus, more white spaces are available for SUs. Consequently, the mean SU delay decreases as $\gamma_{1}=$ $\gamma_{2}$ decreases.

In Figure 5, the throughput $\eta_{\mathrm{PU}}$ of PUs is plotted versus $\lambda_{1}$. An increase in the PU arrival rate results in a smaller increase in the throughput $\eta_{\mathrm{PU}}$ for smaller values of $\gamma_{1}=\gamma_{2}$. This is again due to the increased blocking probability of PUs for smaller $\gamma_{1}=\gamma_{2}$ as shown above (see Figure 3 ).

In Figure 6, the throughput $\eta_{\mathrm{SU}}$ of SUs is given versus $\lambda_{1}$, for different values of $\gamma_{1}=\gamma_{2}$. Obviously, for increasing $\lambda_{1}$, the throughput of SUs decreases regardless of the value of $\gamma_{1}=\gamma_{2}$. When $\lambda_{1}$ is small, it can be seen that the SU throughput is smaller for low $\gamma_{1}=\gamma_{2}$. This is again explained by the fact that, for small values of $\gamma_{1}=\gamma_{2}$, PU arrivals

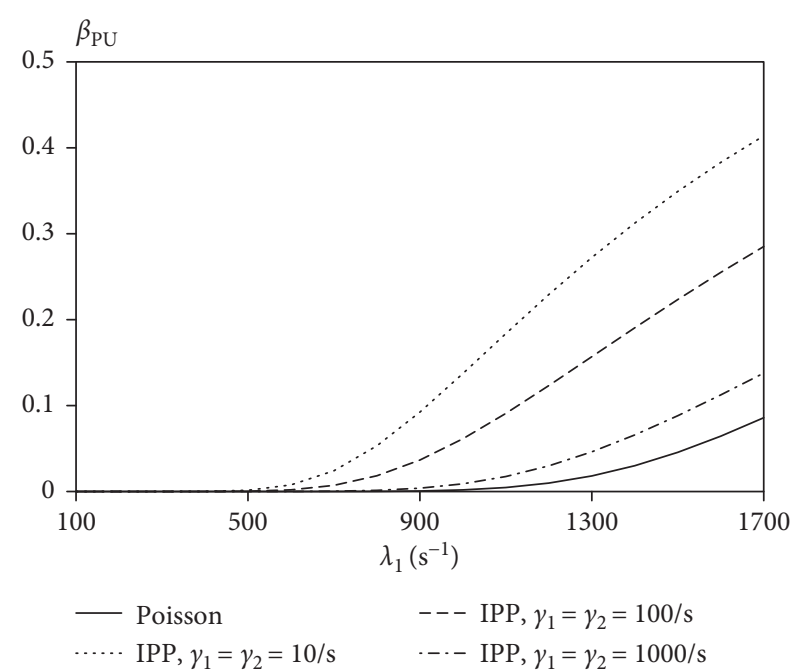

FIGURE 3: Blocking probability $\beta_{\mathrm{PU}}$ of PUs versus the average PU arrival rate $\lambda_{1}, \quad$ for $\rho_{\mathrm{SU}}=0.5, \quad N=20, \quad K=50$, $1 / \sigma=1 / \mu_{1}=1 / \mu_{2}=10 \mathrm{~ms}, \quad p_{f 1}=p_{m 1}=p_{m 2}=0, \delta_{f 2}=0 / \mathrm{s}$, and different values of $\gamma_{1}=\gamma_{2}$.

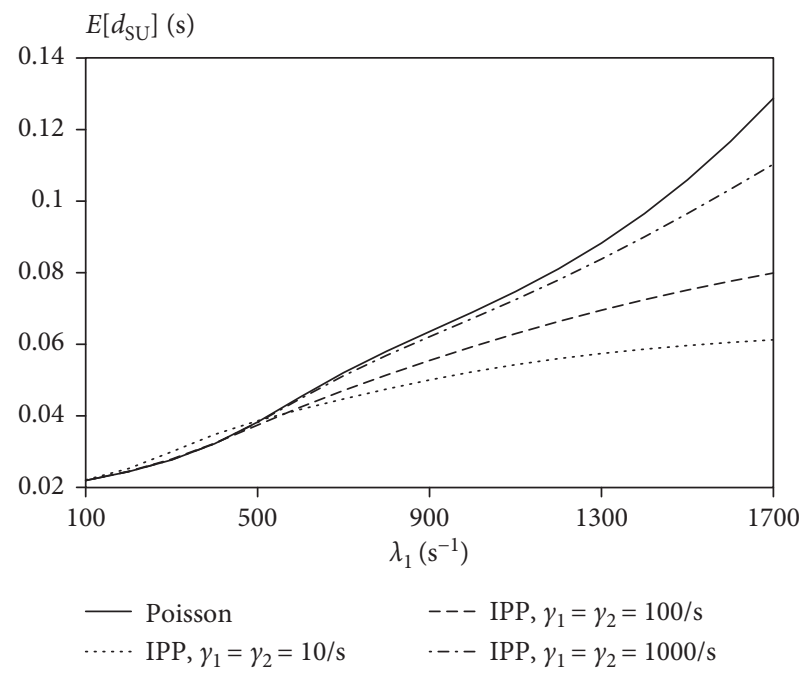

Figure 4: Mean delay $E\left[d_{S U}\right]$ of SUs versus the average PU arrival rate $\lambda_{1}$, for $\rho_{\mathrm{SU}}=0.5, N=20, K=50,1 / \sigma=1 / \mu_{1}=1 / \mu_{2}=10 \mathrm{~ms}$, $p_{f 1}=p_{m 1}=p_{m 2}=0, \delta_{f 2}=0 / \mathrm{s}$, and different values of $\gamma_{1}=\gamma_{2}$.

during active periods occupy more channels, and less white spaces are available for SUs. For a high PU load, the number of PU packets admitted to the system is higher in case of high $\gamma_{1}=\gamma_{2}$ due to a decreased blocking probability of PUs. So, for high $\lambda_{1}$, the effect of $\gamma_{1}=\gamma_{2}$ on the throughput is reversed, i.e., the throughput of SUs increases for decreasing $\gamma_{1}=\gamma_{2}$.

Figure 7 shows the SU blocking probability $\beta_{\mathrm{SU}}$ versus $\lambda_{1}$, for different $\gamma_{1}=\gamma_{2}$. We observe that the SU blocking probability increases as the average PU arrival rate $\lambda_{1}$ increases. For small values of $\lambda_{1}$, the blocking probability $\beta_{\mathrm{SU}}$ is higher when $\gamma_{1}=\gamma_{2}$ is small. For high $\lambda_{1}$ (e.g., $\lambda_{1}=1600 / \mathrm{s}$ ), the order of the curves is reversed. These observations are in full agreement with those of Figure 6. Also, note that, 


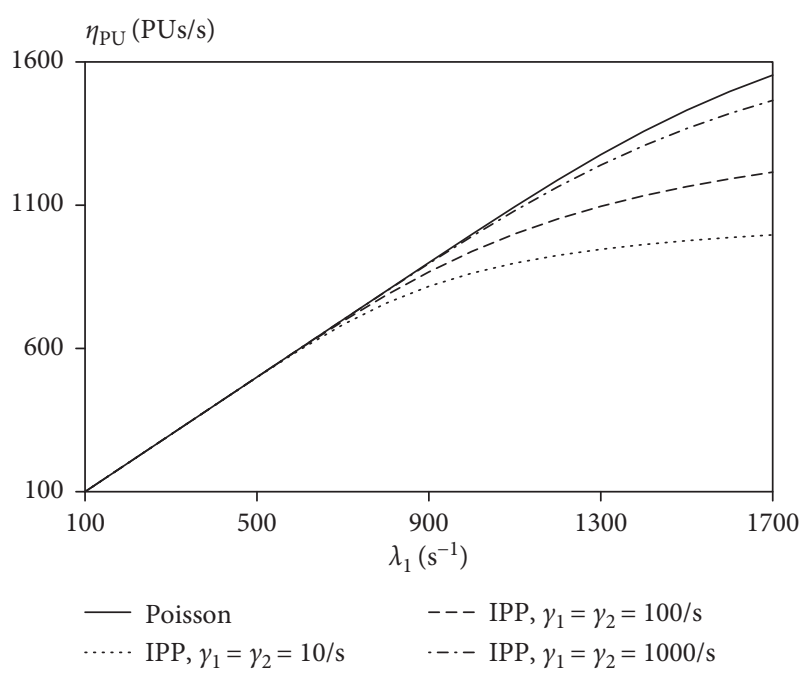

FIGURE 5: Throughput $\eta_{\mathrm{PU}}$ of PUs versus the average PU arrival rate $\lambda_{1}$, for $\rho_{\mathrm{SU}}=0.5, \quad N=20, K=50,1 / \sigma=1 / \mu_{1}=1 / \mu_{2}=10 \mathrm{~ms}$, $p_{f 1}=p_{m 1}=p_{m 2}=0, \delta_{f 2}=0 / \mathrm{s}$, and different values of $\gamma_{1}=\gamma_{2}$.

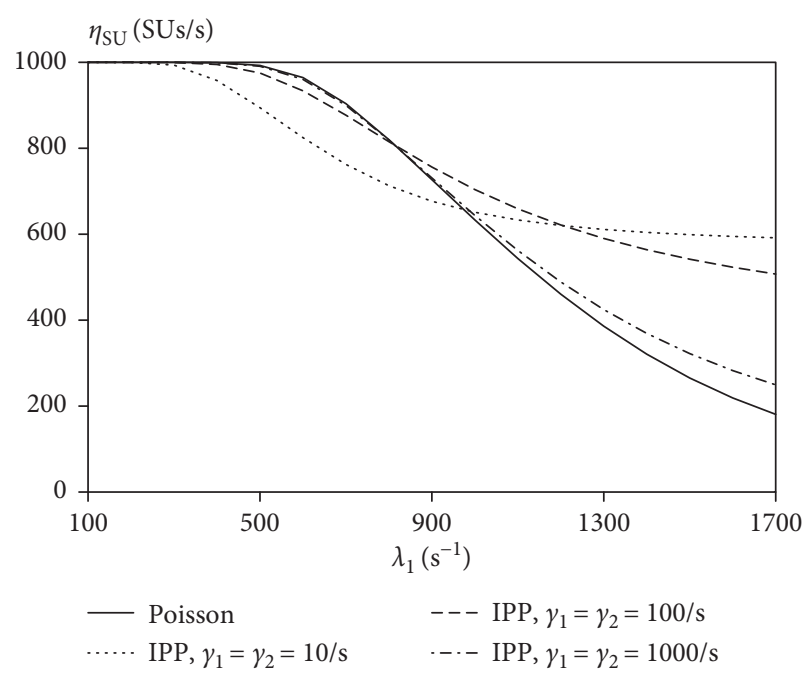

FIGURE 6: Throughput $\eta_{\mathrm{SU}}$ of SUs versus the average PU arrival rate $\lambda_{1}$, for $\rho_{\mathrm{SU}}=0.5, N=20, K=50,1 / \sigma=1 / \mu_{1}=1 / \mu_{2}=10 \mathrm{~ms}$, $p_{f 1}=p_{m 1}=p_{m 2}=0, \delta_{f 2}=0 / \mathrm{s}$, and different values of $\gamma_{1}=\gamma_{2}$.

intuitively, the SU blocking probability is expected to decrease as the maximum number $K$ of sensing SUs increases. A detailed study of the impact of $K$ on the CRN performance under Poisson PU arrivals and perfect spectrum sensing has been performed in [22].

In all the figures above, it can be seen that, for the highest considered value of $\gamma_{1}=\gamma_{2}=1000 / \mathrm{s}$ (when the system switches between the active and inactive states more quickly and the PU arrival process is thus less bursty), the performance measures of PUs and SUs are closest to the case of Poisson arrivals, whereas already indicated burstiness or time correlation is completely absent.

In our next examples, we explore the effect of SU sensing errors on the performance of PUs and SUs when PU packet

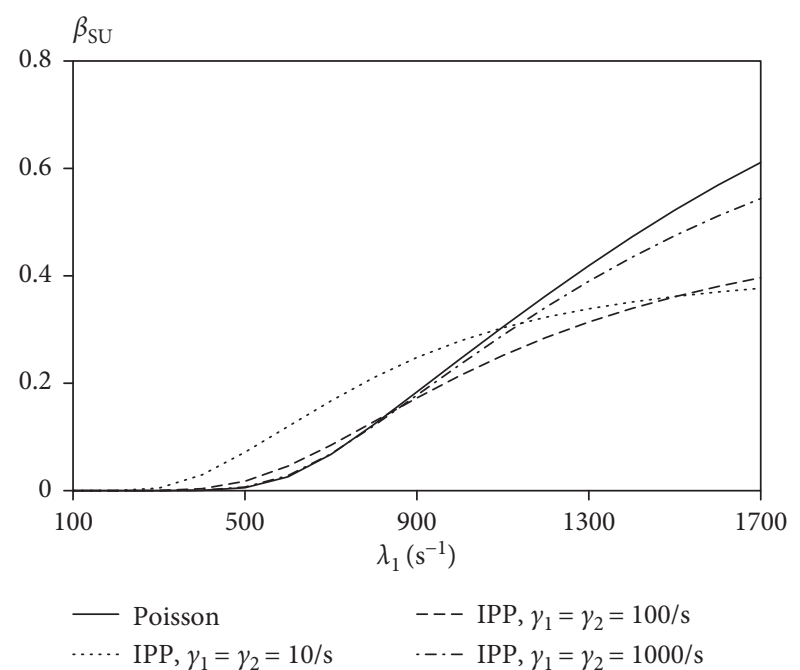

FIGURE 7: Blocking probability $\beta_{\mathrm{SU}}$ of SUs versus the average PU arrival rate $\lambda_{1}, \quad$ for $\rho_{\mathrm{SU}}=0.5, \quad N=20, \quad K=50$, $1 / \sigma=1 / \mu_{1}=1 / \mu_{2}=10 \mathrm{~ms}, \quad p_{f 1}=p_{m 1}=p_{m 2}=0, \quad \delta_{f 2}=0 / \mathrm{s}$, and different values of $\gamma_{1}=\gamma_{2}$.

arrivals are represented by an IPP. We first focus on the impact of the class-A and class-B misdetection probabilities $p_{m 1}$ and $p_{m 2}$ in Figures 8-10. In these figures, we consider an IPP with mean active and inactive periods of $1 / \gamma_{1}=1 / \gamma_{2}=10 \mathrm{~ms}$, i.e., $\gamma_{1}=\gamma_{2}=100 / \mathrm{s}$. We set $p_{f 1}=0$ and $\delta_{f 2}=0 / \mathrm{s}$, which means there are no false alarms.

Figure 8 shows the mean delay $E\left[d_{S U}\right]$ of SUs versus the average PU arrival rate $\lambda_{1}$, for different values of $p_{m 1}=p_{m 2}$. We observe that, for small values of $p_{m 1}$ and $p_{m 2}$, the mean SU delay substantially increases as $\lambda_{1}$ increases. In this case, the number of collisions between PUs and SUs is small (see also Figure 10), and the number of SUs waiting to transmit is large. For higher values of $p_{m 1}$ and $p_{m 2}$, the number of collisions increases; hence, the number of sensing SUs decreases, and an increase of $\lambda_{1}$ has a smaller effect on the mean SU delay.

In Figure 9, the throughput $\eta_{\mathrm{SU}}$ of SUs is plotted versus $\lambda_{1}$, again for different $p_{m 1}=p_{m 2}$. For small values of $\lambda_{1}, \eta_{\mathrm{SU}}$ is larger for smaller $p_{m 1}=p_{m 2}$. For smaller $p_{m 1}=p_{m 2}$, the number of collisions is smaller and, as expected, more SUs can access the channels, leading to a higher throughput. However, a more surprising behavior of the throughput $\eta_{\mathrm{SU}}$ is detected when $\lambda_{1}$ is larger. In particular, when $p_{m 1}=p_{m 2} \leq 0.2$, a slightly larger $\mathrm{SU}$ throughput is observed for larger $p_{m 1}=p_{m 2}$, i.e., $\eta_{\mathrm{SU}}$ is higher even though there are more collisions, which is counterintuitive. In this case, larger $\lambda_{1}$ causes more collisions between PUs and SUs, new white spaces appear that are successfully occupied by SUs, and also $\eta_{\mathrm{SU}}$ increases. This behavior is found to persist regardless of the value of $\gamma_{1}=\gamma_{2}$ and also when the values of the false alarm parameters are not equal to zero. When $p_{m 1}=p_{m 2}>0.2$, the described behavior is found not to exist, and the larger $p_{m 1}=p_{m 2}$, the lower $\eta_{\mathrm{SU}}$.

Figure 10 shows the collision rate $\alpha$ between PUs and SUs versus the average PU arrival rate $\lambda_{1}$, for different $p_{m 1}=p_{m 2}$. From this figure, we notice that, in case PU 


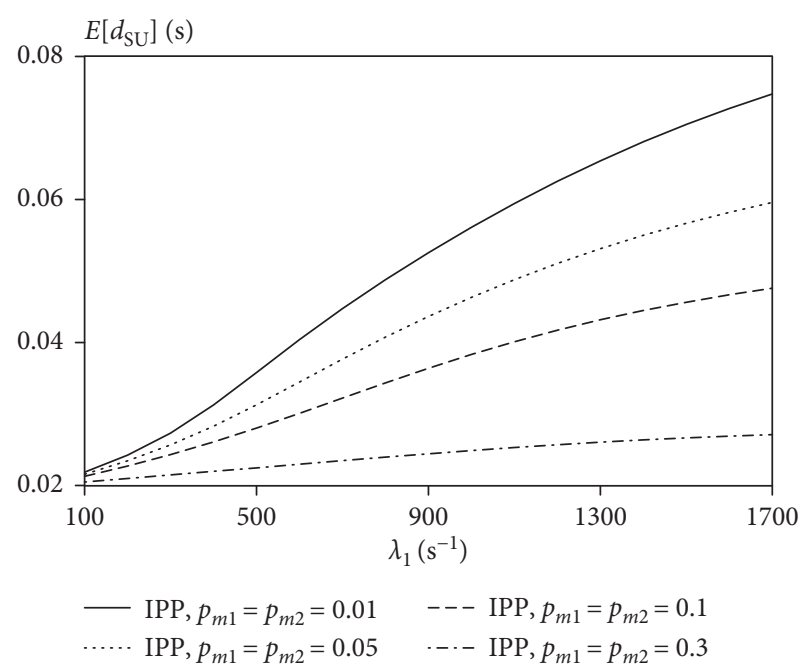

Figure 8: Mean delay $E\left[d_{S U}\right]$ of SUs versus the average PU arrival rate $\lambda_{1}$, for $\rho_{\mathrm{SU}}=0.5, N=20, K=50,1 / \sigma=1 / \mu_{1}=1 / \mu_{2}=10 \mathrm{~ms}$, $1 / \gamma_{1}=1 / \gamma_{2}=10 \mathrm{~ms}, p_{f 1}=0, \delta_{f 2}=0 / \mathrm{s}$, and different values of $p_{m 1}=p_{m 2}$.

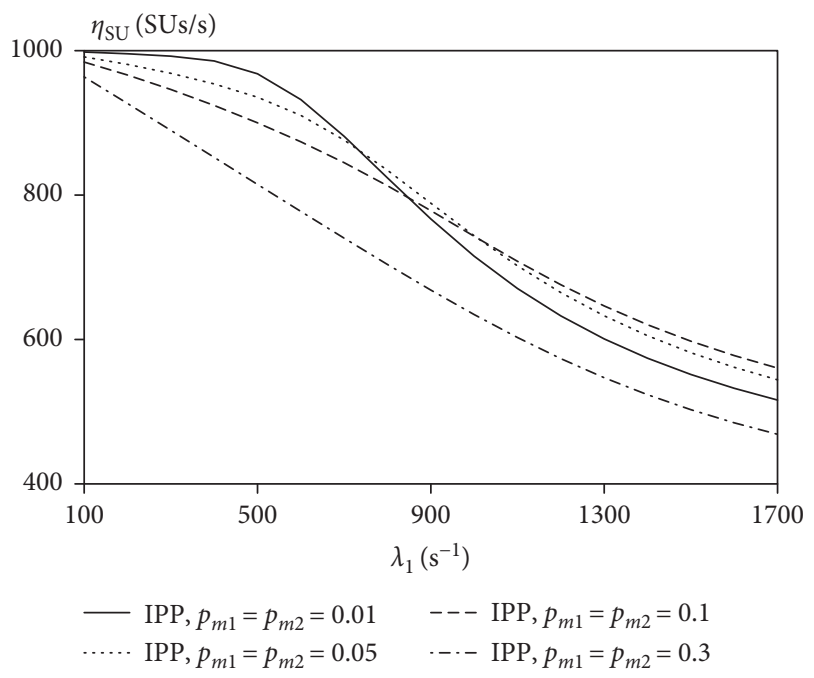

FIGURE 9: Throughput $\eta_{\text {SU }}$ of SUs versus the average PU arrival rate $\lambda_{1}$, for $\rho_{\mathrm{SU}}=0.5, \quad N=20, K=50,1 / \sigma=1 / \mu_{1}=1 / \mu_{2}=10 \mathrm{~ms}$, $1 / \gamma_{1}=1 / \gamma_{2}=10 \mathrm{~ms}, p_{f 1}=0, \delta_{f 2}=0 / \mathrm{s}$, and different values of $p_{m 1}=p_{m 2}$.

arrivals are represented by an IPP, the quality of service of the PU network can be seriously compromised due to the misdetection of PUs by SUs even when $\lambda_{1}$ is small. For instance, for a PU load $\rho_{\mathrm{PU}}=0.1$, i.e., for $\lambda_{1}=200 / \mathrm{s}$, and $p_{m 1}=p_{m 2}=0.1$, the collision rate is around 33 collisions per second for the considered IPP as compared to 23 collisions per second for the case of Poisson arrivals.

Figure 11 shows the collision rate $\alpha$ versus $\lambda_{1}$, for $p_{m 1}=$ $p_{m 2}=0.2$ and different values of $\gamma_{1}=\gamma_{2}$. We again set $p_{f 1}=$ 0 and $\delta_{f 2}=0 / \mathrm{s}$. It can be seen that, for the considered case of $p_{m 1}=p_{m 2}=0.2$, the parameters $\gamma_{1}=\gamma_{2}$ do have an influence on $\alpha$. For values of $p_{m 1}=p_{m 2}<0.2$ and larger values of

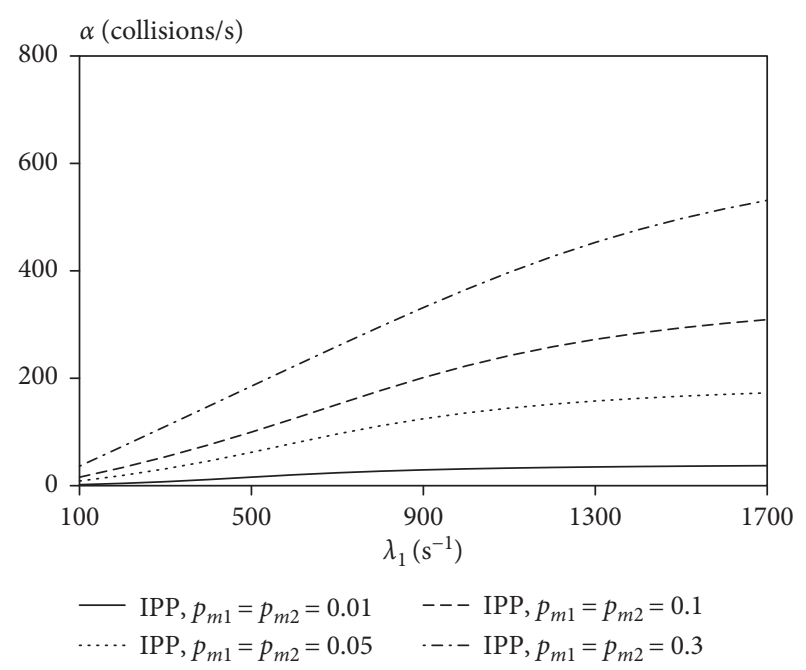

FIgURe 10: Collision rate $\alpha$ between PUs and SUs versus the average PU arrival rate $\lambda_{1}$, for $\rho_{\mathrm{SU}}=0.5, \quad N=20, \quad K=50$, $1 / \sigma=1 / \mu_{1}=1 / \mu_{2}=10 \mathrm{~ms}, 1 / \gamma_{1}=1 / \gamma_{2}=10 \mathrm{~ms}, p_{f 1}=0, \delta_{f 2}=0 / \mathrm{s}$, and different values of $p_{m 1}=p_{m 2}$.

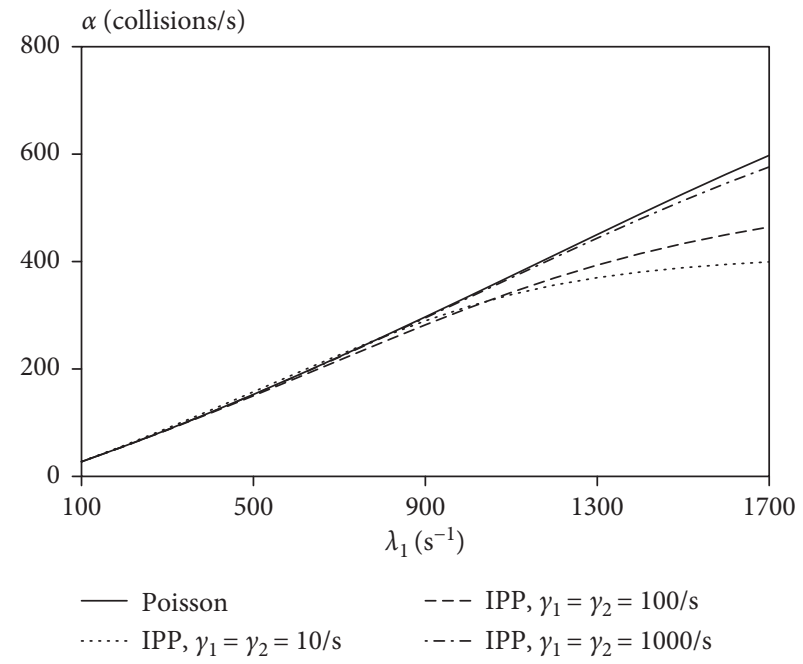

FIgURE 11: Collision rate $\alpha$ between PUs and SUs versus the average PU arrival rate $\lambda_{1}$, for $\rho_{\mathrm{SU}}=0.5, \quad N=20, \quad K=50$, $1 / \sigma=1 / \mu_{1}=1 / \mu_{2}=10 \mathrm{~ms}, \quad p_{m 1}=p_{m 2}=0.2, \quad p_{f 1}=0, \delta_{f 2}=0 / \mathrm{s}$, and different values of $\gamma_{1}=\gamma_{2}$.

$\lambda_{1}$, the influence of $\gamma_{1}=\gamma_{2}$ on $\alpha$ is found to be more significant and can exceed 40 percent. For values of $p_{m 1}=p_{m 2}>0.2$, the influence of $\gamma_{1}=\gamma_{2}$ on $\alpha$ is found to decrease. Finally, it is also found that the false alarm parameters have little influence on the collision rate, as expected.

Next, we investigate the effect of the false alarm parameters $p_{f 1}$ and $\delta_{f 2}$. We now set $p_{m 1}=p_{m 2}=0$, and we consider an average PU arrival rate $\lambda_{1}=1000 / \mathrm{s}$.

Figure 12 shows the throughput $\eta_{\mathrm{SU}}$ of SUs versus $\gamma_{1}=\gamma_{2}$, for different values of $p_{f 1}$ and $\delta_{f 2}$. As expected, for increasing values of $p_{f 1}$ and $\delta_{f 2}$, the throughput $\eta_{\mathrm{SU}}$ 


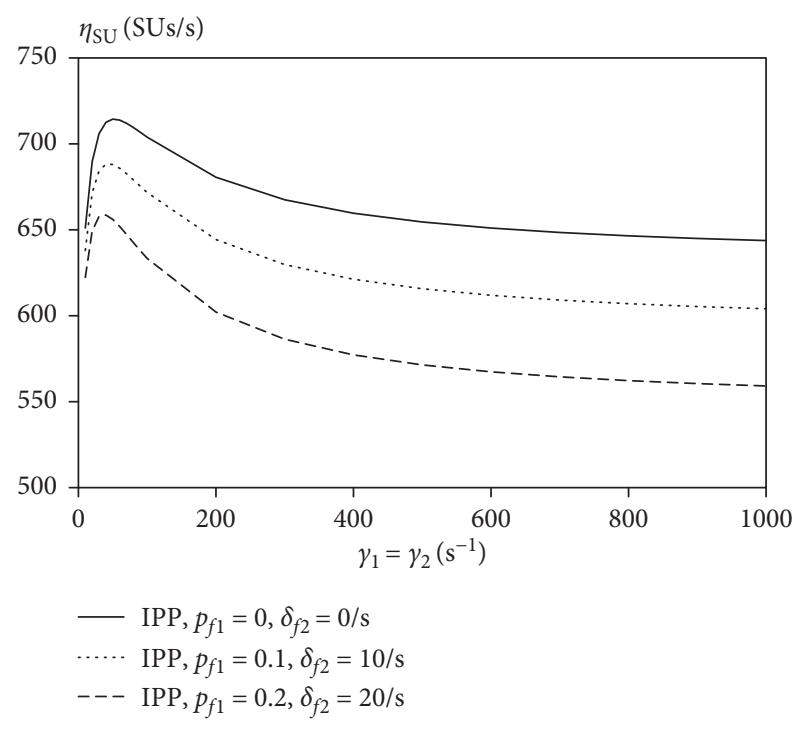

FIGURE 12: Throughput $\eta_{\mathrm{SU}}$ of SUs versus $\gamma_{1}=\gamma_{2}$, for $\rho_{\mathrm{SU}}=0.5$, $N=20, \quad K=50, \quad 1 / \sigma=1 / \mu_{1}=1 / \mu_{2}=10 \mathrm{~ms}, \quad \lambda_{1}=1000 / \mathrm{s}$, $p_{m 1}=p_{m 2}=0$, and different values of $p_{f 1}$ and $\delta_{f 2}$.

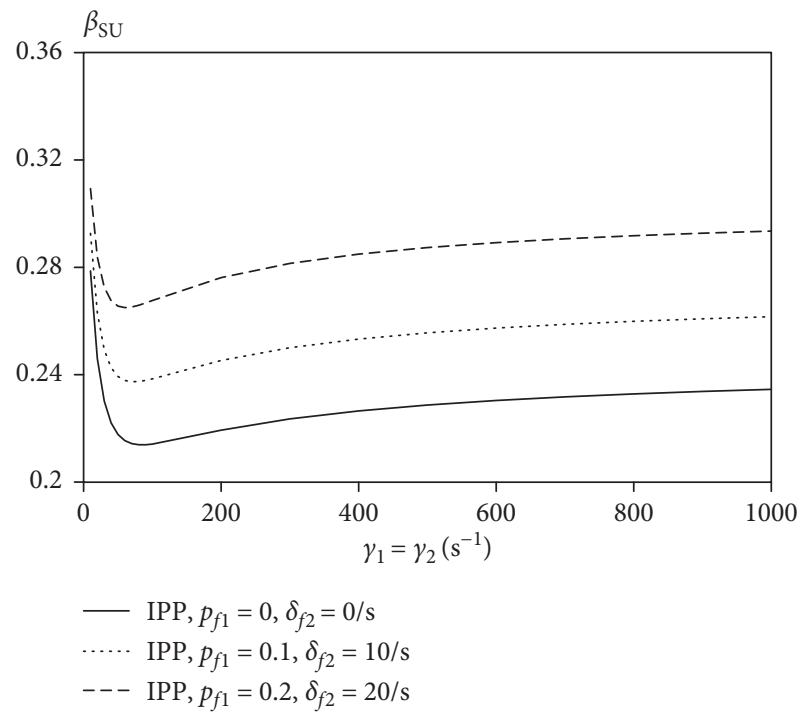

FIGURE 13: Blocking probability $\beta_{\mathrm{SU}}$ of SUs versus $\gamma_{1}=\gamma_{2}$, for $\rho_{\mathrm{SU}}=0.5, N=20, K=50,1 / \sigma=1 / \mu_{1}=1 / \mu_{2}=10 \mathrm{~ms}, \lambda_{1}=1000 / \mathrm{s}$, $p_{m 1}=p_{m 2}=0$, and different values of $p_{f 1}$ and $\delta_{f 2}$.

decreases as more SUs stay in the sensing state. Also, it can be seen that, as $\gamma_{1}=\gamma_{2}$ increases, the throughput $\eta_{\mathrm{SU}}$ first increases and then decreases for higher $\gamma_{1}=\gamma_{2}$. This special effect of $\gamma_{1}=\gamma_{2}$ is also visible in Figure 7 at point $\lambda_{1}=1000 / \mathrm{s}$ for the case $p_{f 1}=0$ and $\delta_{f 2}=0 / \mathrm{s}$ and is due to the considered intermediate value of the PU load. As explained before, for low PU load, $\eta_{\mathrm{SU}}$ increases as $\gamma_{1}=\gamma_{2}$ increases, whereas for high PU load, the effect is reversed.

Figure 13 shows the blocking probability $\beta_{\mathrm{SU}}$ of SUs versus $\gamma_{1}=\gamma_{2}$, for different values of $p_{f 1}$ and $\delta_{f 2}$. The behavior of $\beta_{\mathrm{SU}}$ is clearly in full agreement with that of the SU throughput $\eta_{\mathrm{SU}}$ (see Figure 12). As the values of the false alarm parameters $p_{f 1}$ and $\delta_{f 2}$ increase, the blocking probability $\beta_{\mathrm{SU}}$ decreases.

\section{Conclusion}

In this paper, we have presented a CTMC model to evaluate the performance of cognitive radio networks where SUs can experience sensing errors and PUs arrive according to an interrupted Poisson process. The effect of the IPP parameters and the sensing error parameters on the performance measures of both PU and SU networks has been studied extensively. Future work could look into a further generalization of the model to include time correlation in the SU arrival process as well.

\section{Data Availability}

No data were used to support this study.

\section{Conflicts of Interest}

The authors declare that they have no conflicts of interest.

\section{Acknowledgments}

This work was partially funded by an EOS grant from the Belgian Research Councils FWO and FNRS (grant number 30452698).

\section{References}

[1] J. Mitola and G. Q. Maguire, "Cognitive radio: making software radios more personal," IEEE Personal Communications, vol. 6, no. 4, pp. 13-18, 1999.

[2] K. Kumar, A. Prakash, and R. Tripathi, "Spectrum handoff in cognitive radio networks: a classification and comprehensive survey," Journal of Network and Computer Applications, vol. 61, pp. 161-188, 2016.

[3] F. Khan and K. Nakagawa, "Comparative study of spectrum sensing techniques in cognitive radio networks," in Proceedings Of the 2013 World Congress on Computer and Information Technology, WCCIT 2013, p. 8, Sousse, Tunisia, June 2013.

[4] A. M. Fanan, N. G. Riley, M. Mehdawi, M. Ammar, and M. Zolfaghari, "Survey: a comparison of spectrum sensing techniques in cognitive radio," in Proceedings of the 2014 International Conference on Image Processing, Computers and Industrial Engineering, pp. 65-69, Kuala Lumpur, Malaysia, January 2014

[5] S. Tang, "A general model of opportunistic spectrum sharing with unreliable sensing," International Journal of Communication Systems, vol. 27, no. 1, pp. 31-44, 2014.

[6] J. D. Kim and G. Hwang, "Cross-layer modeling and optimization of multi-channel cognitive radio networks under imperfect channel sensing," Journal of Industrial and Management Optimization, vol. 11, no. 3, pp. 807-828, 2015.

[7] A. Ö. Ercan and M. O. Sunay, "On the feasibility of synchronous, retransmission-based cognitive networks," Ad Hoc Networks, vol. 36, pp. 398-406, 2016.

[8] K. Kulkarni and A. Banerjee, "On stable throughput of cognitive radio networks with cooperating secondary users," IEEE Transactions on Communications, vol. 64, no. 10, pp. 4097-4110, 2016. 
[9] K. Kulkarni and A. Banerjee, "Multi-channel sensing and resource allocation in energy constrained cognitive radio networks," Physical Communication, vol. 23, pp. 12-19, 2017.

[10] J. Jeya, S. S. Kalamkar, and A. Banerjee, "Energy harvesting cognitive radio with channel-aware sensing strategy," IEEE Communications Letters, vol. 18, no. 7, pp. 1171-1174, 2014.

[11] B. Liu, Z. Li, J. Si, and F. Zhou, "Optimal sensing interval in cognitive radio networks with imperfect spectrum sensing," IET Communications, vol. 10, no. 2, pp. 189-198, 2016.

[12] C. Jiang, N. C. Beaulieu, L. Zhang, Y. Ren, M. Peng, and H.-H. Chen, "Cognitive radio networks with asynchronous spectrum sensing and access," IEEE Network, vol. 29, no. 3, pp. 88-95, 2015.

[13] A. Sultana, X. Fernando, and L. Zhao, "An overview of medium access control strategies for opportunistic spectrum access in cognitive radio networks," Peer-to-Peer Networking and Applications, vol. 10, no. 5, pp. 1113-1141, 2017.

[14] Y. Han, E. Ekici, H. Kremo, and O. Altintas, "A survey of MAC issues for TV white space access," Ad Hoc Networks, vol. 27, pp. 195-218, 2015.

[15] I. F. Akyildiz, W.-Y. Lee, and K. R. Chowdhury, "CRAHNs: cognitive radio ad hoc networks," Ad Hoc Networks, vol. 7, no. 5, pp. 810-836, 2009.

[16] P. Venkatesh and H. Sirisena, "Modeling for dimensioning and configuring general packet radio service networks," in Proceedings of the IEEE Wireless Communications and Networking Conference, pp. 1510-1515, Atlanta Georgia, March 2004.

[17] A. Agarwal, S. Dubey, R. Gangopadhyay, and S. Debnath, "Secondary user QoE enhancement through learning based predictive spectrum access in cognitive radio networks," in Proceedings of the 2016 International Conference on Cognitive Radio Oriented Wireless Networks, CrownCom 2016, Grenoble, France, June 2016.

[18] S. M. Ross, Stochastic Processes, John Wiley \& Sons, New York, NY, USA, 2nd edition, 1996.

[19] O. C. Ibe, Markov Processes for Stochastic Modeling, Elsevier, London, UK, 2nd edition, 2013.

[20] R. Durrett, Essentials of Stochastic Processes, Springer, New York, NY, USA, 2nd edition, 2012.

[21] A. S. Zahmati, X. Fernando, and A. Grami, "Steady-state Markov chain analysis for heterogeneous cognitive radio networks," in Proceedings of the 2010 IEEE Sarnoff Symposium, Princeton, NY, USA, April 2010.

[22] O. Salameh, K. De Turck, H. Bruneel, C. Blondia, and S. Wittevrongel, "Analysis of secondary user performance in cognitive radio networks with reactive spectrum handoff," Telecommunication Systems, vol. 65, no. 3, pp. 539-550, 2017.

[23] H.-F. Lin, S.-M. Cheng, and P.-Y. Huang, "Cognitive access in multichannel wireless networks using two-dimension Markov chain," in Proceedings of the 2014 International Wireless Communications and Mobile Computing Conference, pp. 169-173, Nicosia, Cyprus, August 2014.

[24] I. Suliman, J. Lehtomäki, T. Bräysy, and K. Umebayashi, "Analysis of cognitive radio networks with imperfect sensing," in Proceedings of the 20th IEEE International Symposium on Personal, Indoor and Mobile Radio Communications, pp. 1616-1620, Tokyo, Japan, September 2009.

[25] I. M. Suliman, J. J. Lehtomaki, K. Umebayashi, and M. Katz, "Analysis of cognitive radio networks with imperfect sensing," IEICE Transactions on Communications, vol. E96-B, no. 6, pp. 1605-1615, 2013.

[26] B. Couceiro and J. Brito, "Performance evaluation of the opportunistic spectrum access in a cognitive radio network with imperfect sensing," in Proceedings of the Fifteenth International Conference on Networks, ICN 2016, pp. 78-83, Lisbon, Portugal, February 2016.

[27] O. Altrad, S. Muhaidat, A. Al-Dweik, A. Shami, and P. D. Yoo, "Opportunistic spectrum access in cognitive radio networks under imperfect spectrum sensing," IEEE Transactions on Vehicular Technology, vol. 63, no. 2, pp. 920-925, 2014.

[28] S. Tang and Y. Xie, "Performance analysis of unreliable sensing for an opportunistic spectrum sharing system," International Journal of Communication Networks and Information Security, vol. 3, no. 3, pp. 240-246, 2011.

[29] O. Salameh, K. De Turck, D. Fiems, H. Bruneel, and S. Wittevrongel, "Performance analysis of a cognitive radio network with imperfect spectrum sensing," IEICE Transactions on Communications, vol. E101-B, no. 1, pp. 213-222, 2018.

[30] I. M. Suliman, J. Lehtomäki, and K. Umebayashi, "On the effect of false alarm rate on the performance of cognitive radio networks," EURASIP Journal on Wireless Communications and Networking, vol. 2015, no. 1, 2015.

[31] Y. Lu, D. Wang, and M. Fattouche, "Cooperative spectrumsensing algorithm in cognitive radio by simultaneous sensing and BER measurements," EURASIP Journal on Wireless Communications and Networking, vol. 2016, no. 1, 2016.

[32] N. Tadayon and S. Aissa, "Multi-channel cognitive radio networks: modeling, analysis and synthesis," IEEE Journal on Selected Areas in Communications, vol. 32, no. 11, pp. 20652074, 2014.

[33] W. Afifi and M. Krunz, "Exploiting self-interference suppression for improved spectrum awareness/efficiency in cognitive radio systems," in Proceedings of the 2013 IEEE International Conference on Computer Communications, pp. 1258-1266, Turin, Italy, April 2013.

[34] S. A. AlQahtani and H. Ahmed, "An admission control scheme for secondary users in cognitive radio networks," in Proceedings of the IEEE 11th International Conference on Networking, Sensing and Control, ICNSC 2014, pp. 245-250, Miami, FL, USA, April 2014.

[35] S. L. Castellanos-Lopez, G. Hernandez-Valdez, M. E. RiveroAngeles, and F. A. Cruz-Perez, "Call admission control strategy for cognitive radio networks with VoIP-traffic," in Proceedings of the IEEE 81st Vehicular Technology Conference, VTC Spring 2015, p. 7, Glasgow, Scotland, May 2015.

[36] K. R. Chowdhury, M. Di Felice, and I. F. Akyildiz, "TPCRAHN: a transport protocol for cognitive radio ad-hoc networks," in Proceedings of the 2009 IEEE International Conference on Computer Communications, pp. 2482-2490, Rio de Janeiro, Brazil, April 2009.

[37] C. R. Baugh, J. Huang, R. Schwartz, and D. Trinkwon, "Traffic model for 802.16 TG3 MAC/PHY simulations," Technical Report 802.16.3c-01/30r1, p. 26, IEEE, Piscataway, NJ, USA, 2001. 